\title{
Unsteady Heat-Flux Measurements of Second-Mode Instability Waves in a Hypersonic Boundary Layer
}

\author{
Michael A. Kegerise* and Shann J. Rufer ${ }^{\dagger}$ \\ NASA Langley Research Center, Hampton, VA 23681
}

\begin{abstract}
In this paper we report on the application of the atomic layer thermopile (ALTP) heatflux sensor to the measurement of laminar-to-turbulent transition in a hypersonic flat plate boundary layer. The centerline of the flat-plate model was instrumented with a streamwise array of ALTP sensors and the flat-plate model was exposed to a Mach 6 freestream over a range of unit Reynolds numbers. Here, we observed an unstable band of frequencies that are associated with second-mode instability waves in the laminar boundary layer that forms on the flat-plate surface. The measured frequencies, group velocities, phase speeds, and wavelengths of these instability waves are in agreement with data previously reported in the literature. Heat flux time series, and the Morlet-wavelet transforms of them, revealed the wave-packet nature of the second-mode instability waves. In addition, a laser-based radiative heating system was developed to measure the frequency response functions (FRF) of the ALTP sensors used in the wind tunnel test. These measurements were used to assess the stability of the sensor FRFs over time and to correct spectral estimates for any attenuation caused by the finite sensor bandwidth.
\end{abstract}

\section{Nomenclature}

a wavelet scale

$c_{g} \quad$ group velocity

$c_{p} \quad$ phase speed

voltage fluctuation from photodiode or ALTP sensor

$E \quad$ DC-coupled voltage from ALTP sensor

$E_{o} \quad$ offset voltage from ALTP sensor amplifier

$f \quad$ frequency

$G_{A C} \quad$ gain of AC-coupled signal

$G_{D C} \quad$ gain of DC-coupled signal

$G_{e e} \quad$ auto-spectral density of photodiode voltage fluctuations

$G_{e q} \quad$ cross-spectral density between photodiode voltage fluctuations and heat-flux fluctuations

$G_{q q} \quad$ auto-spectral density of heat-flux fluctuations

$H \quad$ frequency response function

$i \quad$ ALTP sensor port-number index

$M_{e} \quad$ Mach number at edge of boundary layer

$Q \quad$ DC-coupled heat flux signal from ALTP sensor

$q \quad$ heat-flux fluctuation

$R \quad \sqrt{R e_{x}}$

Re unit Reynolds number based on freestream conditions

$R e_{x} \quad$ Reynolds number based on conditions at boundary-layer edge and the streamwise position

$R_{q_{i} q_{i}} \quad$ auto-correlation function for heat-flux sensor at port number $i$

$R_{q_{i} q_{i+1}}$ cross-correlation function between heat-flux sensors at ports numbers $i$ and $i+1$

$S \quad$ static sensitivity of ALTP sensor

$T_{d} \quad$ time delay

${ }^{*}$ Research Scientist, Flow Physics and Control Branch, M.S. 170. Senior Member AIAA.

${ }^{\dagger}$ Research Scientist, Aerothermodynamics Branch, M.S. 408A. Senior Member AIAA. 


$\begin{array}{ll}u_{e} & \text { streamwise velocity at edge of boundary layer } \\ W & \text { continuous wavelet transform } \\ x & \text { streamwise position } \\ \alpha & \text { model angle of attack } \\ \delta & \text { boundary-layer thickness } \\ \Delta x & \text { streamwise spacing between adjacent heat-flux sensors } \\ \phi & \text { phase angle } \\ \Psi & \text { Morlet-wavelet function } \\ \rho & \text { cross-correlation coefficient } \\ \tau & \text { lag time in correlation functions or wavelet time delay } \\ (\overline{)} & \text { mean value }\end{array}$

\section{Introduction}

I AmINAR-to-turbulent boundary-layer transition is of critical concern for the design of many hypersonic

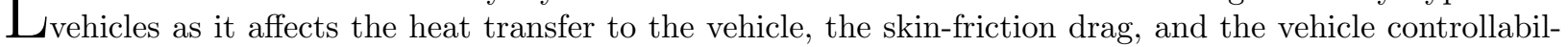
ity. Although our understanding of the physical mechanisms that cause transition in hypersonic boundary layers has greatly improved over the past several decades, our current ability to predict when and where transition will occur on a given hypersonic configuration is still lacking. In the near term, improvements to our predictive methodologies will depend greatly on both computational methods and experimental measurements in ground-based facilities. Experimental measurements, with unsteady or dynamic sensors in particular, provide critical validation data for boundary layer stability and transition calculations and they allow us to identify the mode(s) of boundary-layer transition for a given hypersonic configuration. Unsteady measurements can also be used to evaluate various methods for boundary layer transition control.

The flow environment in a transitional hypersonic boundary layer presents many challenges for unsteady instrumentation. Intrusive instruments, such as hot-wire anemometers, or surface-based dynamic sensors must survive the high-temperature and high-enthalpy conditions common to hypersonic flows. For scalemodel tests in ground-based facilities, where the boundary-layer thickness on the model is small (on the order of $\mathrm{mm})$, unsteady sensors must have high spatial resolution, high bandwidth $(\sim 1 \mathrm{MHz})$, and high sensitivity to resolve the instability waves associated with transition. Very few of our current unsteady measurement techniques can satisfy these requirements, and each one that is available has both strengths and weaknesses. Hot-wires, for example, have been used in several studies ${ }^{1-4}$ to perform off-body measurements of instability waves, but these measurements are limited to lower hypersonic Mach numbers where the temperature or enthalpy is low enough to ensure survival of the hot-wire sensor. In addition, hot wires often have insufficient bandwidth to resolve the instability waves of interest and calibration of hot wires is difficult in a hypersonic flow. Non-intrusive, off-body measurement techniques, such as focused schlieren deflectometry ${ }^{5}$ and focused laser differential interferometry ${ }^{6}$ are certainly more promising since there are no issues with the high-temperature, high-enthalpy environments. In addition, the bandwidths of these systems can be very high $(>1 \mathrm{MHz})$, being limited only by the bandwidth of the photo detectors that are utilized. However, these techniques do require a high-degree of optical access to the flow field and can have poor spatial resolution in the flow spanwise direction. Fast-response piezoelectric pressure transducers have been utilized in a large number of studies for measurements of the surface pressure fluctuations induced by transitional hypersonic boundary layers. ${ }^{7-10}$ These transducers offer a bandwidth on the order of $1 \mathrm{MHz}$, but they are difficult to calibrate and may suffer from spatial averaging. More recently, a new class of fast-response heat-flux sensors, the Atomic Layer Thermopile (ALTP), was applied to the measurement of surface heat-flux fluctuations induced by transitional hypersonic boundary layers. ${ }^{11-15}$ These sensors are reported to have a bandwidth of $\sim 1 \mathrm{MHz}$, a spatial resolution of $1 \mathrm{~mm}^{2}$, and a linear static response from milliwatts to kilowatts per square centimeter. ${ }^{16}$ Being a surface-based measurement, however, these sensors can provide only a partial picture of the transition process. Nevertheless, particularly in cases where off-body unsteady measurements are not feasible, the ALTP sensors are an attractive option for the study of hypersonic boundary layer transition.

In the present work, our objective was to gain experience with the ALTP sensors for transition measurements in our conventional hypersonic blow-down tunnels at NASA Langley Research Center. As a test bed, we instrumented the centerline of a flat plate model with a streamwise array of ALTP sensors and exposed 


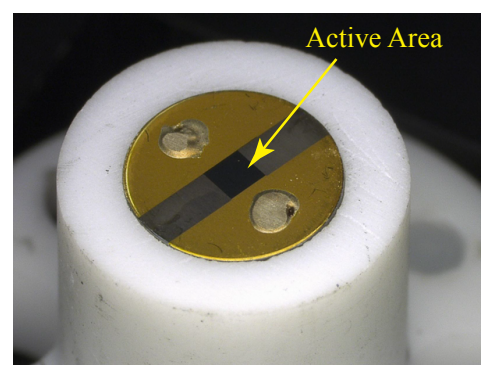

(a) Perspective view at 30X optical magnification.

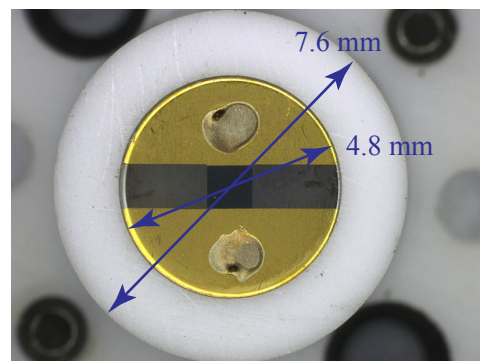

(b) Top view at $30 \mathrm{X}$ optical magnification.

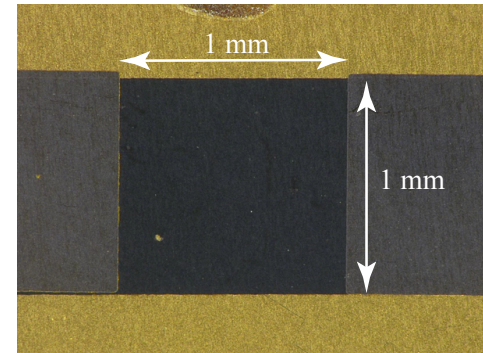

(c) Close-up image of sensor active area at $150 \mathrm{X}$ optical magnification.

Figure 1. Close-up images of an atomic layer thermopile (ALTP) heat-flux sensor.

the model to a Mach 6 freestream over a range of unit Reynolds numbers. Here, we measured the growth and evolution of second-mode instability waves in the laminar boundary layer that forms on the flat plate model surface. In addition, we developed a laser-based system for the dynamic calibration of the ALTP sensors. Pre- and post-test measurements of the frequency response function allowed us to check the stability of the sensor dynamic response over time and to correct spectral estimates for any attenuation caused by the finite sensor bandwidth. In this paper, we will report the details of the flat plate transition measurements and the results of the dynamic calibrations.

\section{Experimental Setup}

The experimental measurements were performed in the NASA Langley Aerothermodynamics Laboratory 20-Inch Mach 6 Air Tunnel. ${ }^{17}$ This wind tunnel is a blowdown facility in which heated, dried, and filtered air is used as the test gas. The tunnel has a two-dimensional, contoured nozzle that opens into a $520.7 \mathrm{~mm}$ by $508 \mathrm{~mm}$ test section. The tunnel is equipped with a bottom-mounted injection system that can transfer the model from the sheltered model box to the tunnel centerline in approximately 1.5 seconds. Run times on the order of 20 minutes are possible, although for the current study, we utilized run times of about 5 seconds. The nominal reservoir conditions of the facility are stagnation pressures of $206.8 \mathrm{kPa}$ to $3275 \mathrm{kPa}$ with stagnation temperatures of $422.2 \mathrm{~K}$ to $555.6 \mathrm{~K}$, which very nearly produces perfect gas $(\gamma=1.4)$ freestream flows with Mach numbers between 5.8 and 6.1 and unit Reynolds numbers of $1.64 \times 10^{6} \mathrm{~m}^{-1}$ to $24.0 \times 10^{6} \mathrm{~m}^{-1}$. Recently, measurements of the freestream total pressure fluctuations were performed in the facility, ${ }^{18}$ and they were found to be $1.5 \%$ at a unit Reynolds number of $4.9 \times 10^{6} \mathrm{~m}^{-1}$ and decreased to $1 \%$ as the unit Reynolds number was increased to $23 \times 10^{6} \mathrm{~m}^{-1}$

The test model for our study was a large flat-plate model, $71.12 \mathrm{~cm}$ long by $27.94 \mathrm{~cm}$ wide. The leading edge of the flat plate was sharp with a $15^{\circ}$ wedge angle on the bottom surface of the model. An insert on the model centerline provided 16 locations for sensor installation. The first sensor port was located $21 \mathrm{~cm}$ from the model leading edge and subsequent sensor ports were spaced equally with a $2.79 \mathrm{~cm}$ spacing. For any given test run, four ALTP sensors were installed in the model and the remaining sensor ports were plugged.

The ALTP sensors were manufactured by ForTech HTS GmbH, and close-up photos of a sensor are shown in Fig. 1. The sensors were housed in a Macor insert with an outer diameter of $7.6 \mathrm{~mm}$. The active area of the heat-flux sensor was $1 \mathrm{~mm}^{2}$ and the nominal bandwidth, as reported by the manufacturer, was approximately $620 \mathrm{kHz}$. The nominal static sensitivity for the sensors was $48.0 \mu \mathrm{V} / \mathrm{W} / \mathrm{cm}^{2}$. The output signal from an ALTP sensor was fed to a miniature low-noise voltage amplifier that was placed inside the model to minimize cable length and electronic noise interference. The amplifier provided two output signals, both of which were utilized during the test. One output was an AC-coupled signal with a fixed gain of 5000 and a bandwidth from $17 \mathrm{~Hz}$ to $1 \mathrm{MHz}$. The other output was a DC-coupled signal with an adjustable gain (from 100 to 800 times) and a bandwidth of $100 \mathrm{kHz}$. Further details on the ALTP sensor construction, operating principle, and methods for calibration can be found in Knauss et al. ${ }^{12}$ and Roediger. ${ }^{14}$

Time-series data were acquired simultaneously for all four ALTP sensors that were installed in the model for any given run. The AC-coupled signals were first anti-alias filtered through a 6th-order low-pass filter with a $900 \mathrm{kHz}$ cutoff frequency. The signals were then sampled with a 16-bit A-to-D at 2 million samples 


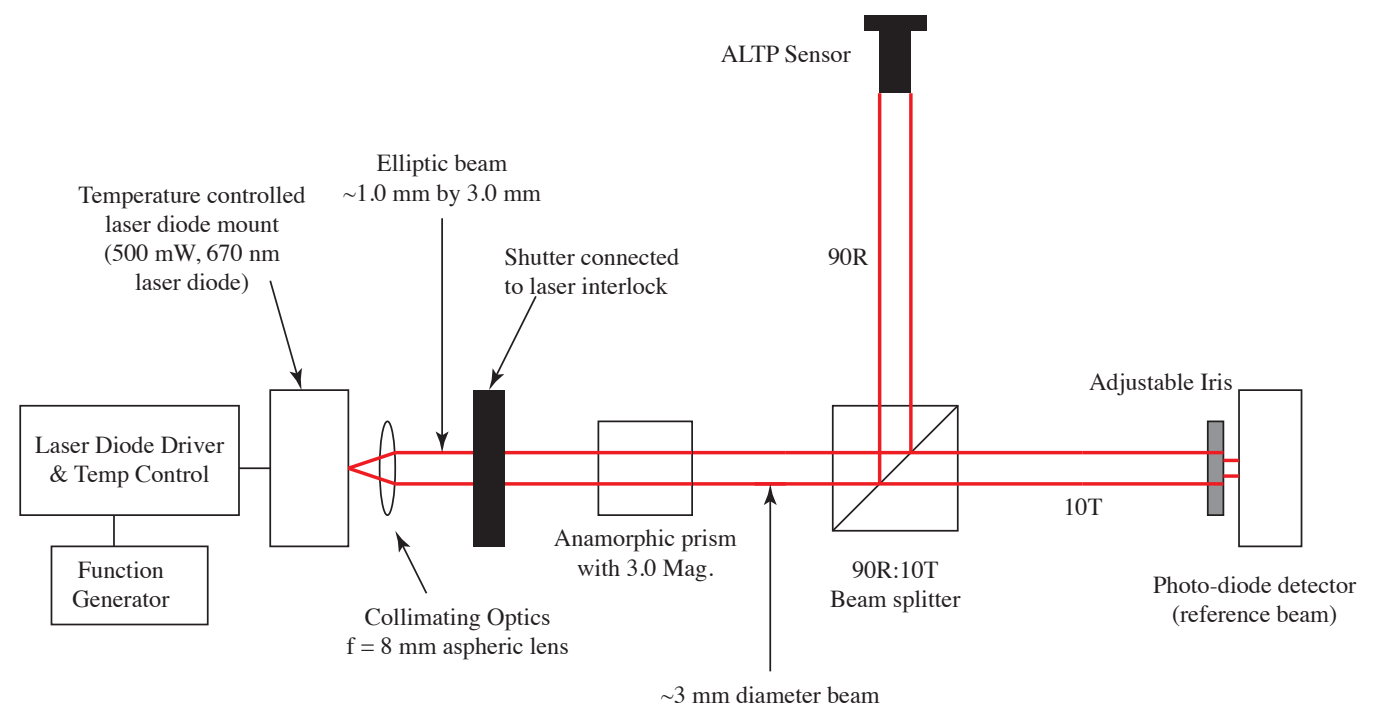

Figure 2. Experimental setup used to measure the ALTP frequency response functions.

per second for 3 seconds. The DC-coupled signals were low-pass filtered with an 8th-order low-pass filter with a $30 \mathrm{kHz}$ cutoff frequency. Those signals were then sampled with a 16-bit A-to-D at 100,000 samples per second for 3 seconds.

Prior to the wind tunnel test, the frequency response functions (FRF) of the ALTP sensors were measured via laser-based radiative heating. This method was previously used to measure the FRF of ALTP sensors ${ }^{12,14,16}$ and is derived from similar setups used to measure the frequency response function of hotwire anemometers. ${ }^{19,20}$ The idea is to radiatively heat the sensor with a laser that is amplitude modulated over a range of frequencies that cover the bandwidth of the sensor. In this way, both the magnitude and the phase of the frequency response function can be determined. A schematic of the setup for the FRF measurements is shown in Fig. 2. The radiative heat flux input was provided by a laser diode with a maximum continuous wave output power of $500 \mathrm{~mW}$ and a wavelength of $670 \mathrm{~nm}$. The laser diode was installed in a temperature-controlled mount that provides precise temperature control of the laser diode via a thermoelectric cooler. A laser diode driver was used to operate the laser diode in a constant-current mode and a temperature controller for the thermoelectric cooler was used to maintain the laser-diode package at a constant temperature of $25^{\circ} \mathrm{C}$. Amplitude modulation of the laser output was achieved by modulating the drive current to the laser diode. A function generator, which was input to the laser diode driver circuit, provided the modulation signal. The laser light emitted from the laser diode was collimated by an aspheric lens with an $8 \mathrm{~mm}$ focal length. Since the beam divergence angles parallel and perpendicular to the laser emitting area are different, the collimated beam is elliptical. Therefore an anamorphic prism was used to magnify the minor axis of the laser beam to produce a roughly circular $3 \mathrm{~mm}$ diameter laser beam. The laser light was then directed to a non-polarizing beam splitter that reflected $90 \%$ of the laser-beam energy toward the sensor, and transmitted the remaining $10 \%$ toward a reference photodiode. Here, the photodiode serves as a measure of the radiant heat flux input to the ALTP sensor. Since the bandwidth of the photodiode sensor is on the order of $500 \mathrm{MHz}$, it is expected to have a flat frequency response with negligible phase lag in the frequency band of interest $(\leq 1 \mathrm{MHz})$. In that case, it provides a representation of the heat-flux input to the ALTP sensor that is free of any amplitude attenuation or phase lags.

To determine the ALTP frequency response function with this setup, time-series data for the photodiode and the ALTP sensor were acquired for sine-wave frequencies ranging from $100 \mathrm{~Hz}$ to $900 \mathrm{kHz}$. Here, the time series were acquired at 2 million samples per second for 2 seconds. Sample time series at two different sinewave frequencies are shown in Fig. 3. For each sine-wave frequency, the frequency response was calculated as:

$$
H(f)=G_{e q}(f) / G_{e e}(f),
$$

where $G_{e e}(f)$ is the auto-spectral density of the photodiode signal and $G_{e q}(f)$ is the cross-spectral density 


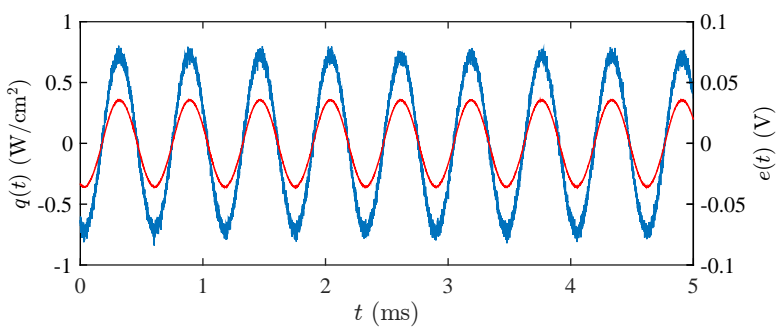

(a) Response for a modulation frequency of $f=1743 \mathrm{~Hz}$.

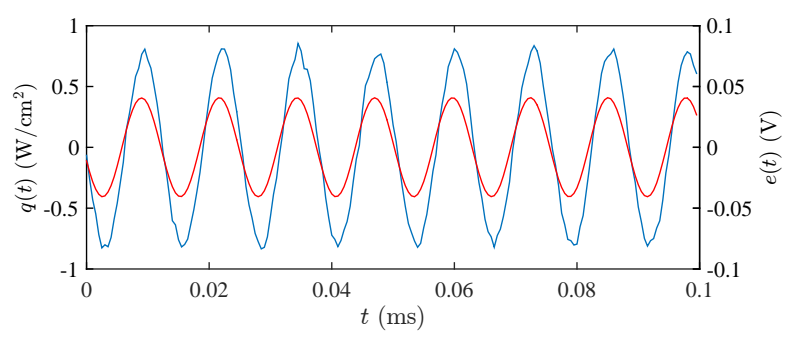

(b) Response for a modulation frequency of $f=78.8 \mathrm{kHz}$.

Figure 3. Response of the ALTP sensor (blue lines) and the reference photodiode (red lines) to a sinusoidally modulated radiant heat flux input.
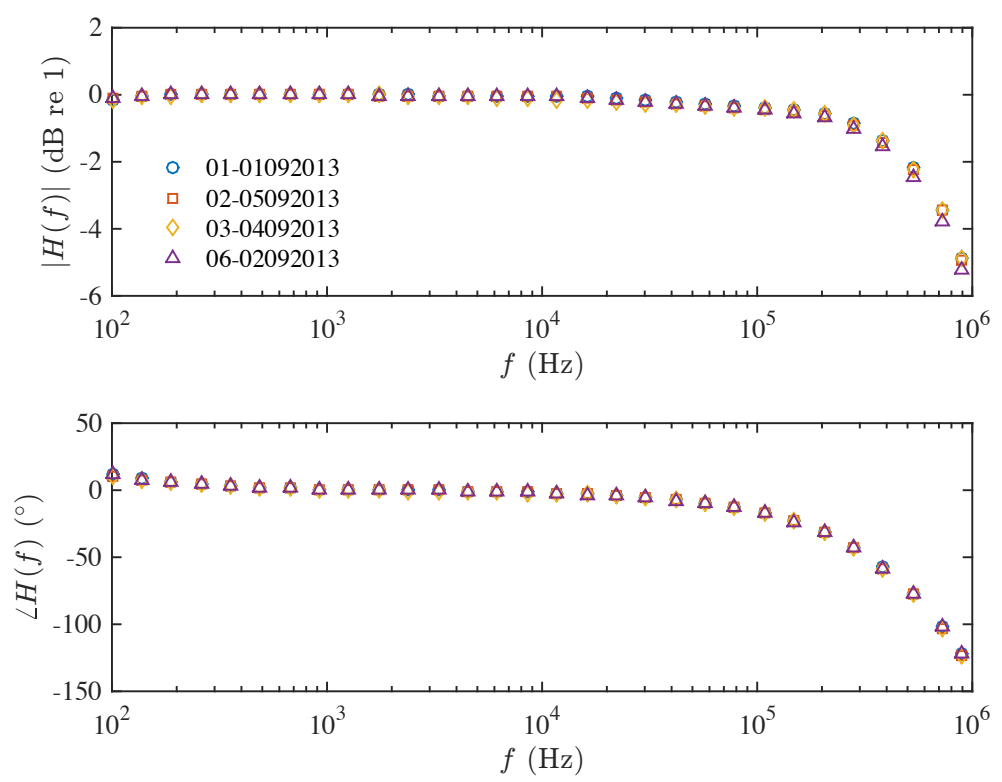

Figure 4. Measured frequency response functions (magnitude and phase) for the four ALTP sensors used in the current study. The legend denotes the sensor serial numbers.

between the photodiode signal and the ALTP signal. Both the auto-spectral and cross-spectral densities were estimated via the Welch method with a block size of 50000 samples, $50 \%$ overlap, and a Hanning window. A total of 160 block averages were performed and the frequency resolution of the estimates were $\Delta f=40 \mathrm{~Hz}$. It is important to note that the reference photodiode does not provide an absolute amplitude of the radiant heat flux input to the ALTP sensor, and so this calculation provides a measure of the ALTP FRF only to within a multiplicative constant. Therefore, when presenting the results, the frequency response functions of the ALTP sensors were normalized by the magnitude of the FRF at the lowest measured frequency. It was then assumed that the true magnitude of the FRF at this frequency was equal to the static sensitivity of the sensors as provided by the manufacturer.

Pre-test measurements of the frequency response magnitude and phase for all four ALTP sensors used in the current study are shown in Fig. 4. For this batch of sensors, the frequency response functions are nearly the same $\left(|H(f)|\right.$ within $\pm 0.36 \mathrm{~dB}$ and $\angle H(f)$ within $\left.\pm 1.3^{\circ}\right)$, with a flat frequency response up to the $-3 \mathrm{~dB}$ cutoff frequency of approximately $650 \mathrm{kHz}$. Post-test measurements of the ALTP sensor FRFs were found to be essentially the same as the pre-test measurements $\left(|H(f)|\right.$ within $\pm 0.27 \mathrm{~dB}$ and $\angle H(f)$ within $\left.\pm 0.3^{\circ}\right)$, indicating that the dynamic response of the sensors was stable over the duration of the test period. 


\section{Data Analysis Methods}

In this section, we present the data-processing methods used to analyze the ALTP sensor time series that were acquired during the wind-tunnel test. Since the response of the ALTP sensor is linear, the mean heat-flux was calculated as:

$$
\bar{Q}=\left(\bar{E}-\overline{E_{o}}\right) /\left(G_{D C} S\right)
$$

where $\bar{E}$ is the average voltage from the DC coupled times series, $G_{D C}$ is the combined gain of the amplifier and filter, and $S$ is the static sensitivity of the sensor as provided by the manufacturer. The voltage, $\overline{E_{o}}$, is the mean offset voltage measured before each run, under vacuum, where the convective heat-flux to the sensor is zero. The instantaneous heat flux fluctuations were calculated as:

$$
q(t)=e(t) /\left(G_{A C} S\right),
$$

where $e(t)$ is the $\mathrm{AC}$ coupled time series voltage, $G_{A C}$ is the combined gain of the amplifier and filter, and $S$ is again the static sensitivity of the sensor.

Using the instantaneous heat flux time series, auto-spectral densities were estimated using the Welch method with blocks of 8192 points, $50 \%$ overlap, and a Hanning window. A total of 1464 block averages were performed and the frequency resolution of the auto-spectral estimates was $244.14 \mathrm{~Hz}$. Due to the finite bandwidth of the ALTP sensors, the auto-spectral density estimates were attenuated at high frequencies (near the cutoff frequency of the sensor and beyond). To account for that attenuation, the auto-spectral densities were corrected according to:

$$
\left.G_{q q}(f)\right|_{\text {corr }}=\left.G_{q q}(f)\right|_{\text {meas }} /|H(f)|^{2},
$$

where $|H(f)|$ is the magnitude of the ALTP frequency response function, as measured via the method described in the previous section.

Cross-spectral densities between adjacent sensor pairs were also estimated with the Welch method using the same parameters as for the auto-spectral density estimates. The cross-spectral densities were then used to develop estimates of the phase speed between adjacent sensors. To that end, the phase angle from the cross-spectral density was defined as:

$$
\phi_{q_{i} q_{i+1}}(f)=\angle G_{q_{i} q_{i+1}}(f),
$$

where $G_{q_{i} q_{i+1}}(f)$ is the cross spectral density between the heat flux time series at sensor ports $i$ and $i+1$. Next, the time delay between the sensors was calculated by:

$$
\left.T_{d}(f)\right|_{q_{i} q_{i+1}}=\phi_{q_{i} q_{i+1}}(f) /(2 \pi f) .
$$

The average phase speed between the sensor pairs was then calculated as:

$$
\left.c_{p}(f)\right|_{q_{i} q_{i+1}}=\Delta x_{q_{i} q_{i+1}} /\left.T_{d}(f)\right|_{q_{i} q_{i+1}},
$$

where $\Delta x_{q_{i} q_{i+1}}$ is the distance between the sensors at ports $i$ and $i+1$. In this phase-speed calcuation, no corrections for the finite FRF of the ALTP sensors were applied. This is allowable because the relative phase differences between the FRFs of the ALTP sensors were found to be nearly zero, as shown in the previous section. In that case, the measured phase differences are due solely to the convection of disturbances from one sensor to another.

To study the time evolution of the frequency content in the unsteady heat-flux signals, the continuous wavelet transform was employed. The continuous wavelet transform is defined as: ${ }^{21}$

$$
W(\tau, a)=\int_{-\infty}^{\infty} q(t) \Psi_{a, \tau}^{*}(t) d t,
$$

where $\Psi_{a, \tau}(t)$ is the wavelet function and $*$ denotes the complex conjugate. This transform projects the signal, $q(t)$, onto all scaled and translated versions of a single mother wavelet. The Morlet wavelet was chosen as the wavelet function for the analysis, and the mother wavelet is defined as:

$$
\Psi(t)=e^{j \omega_{\Psi} t} e^{-t^{2} / 2},
$$




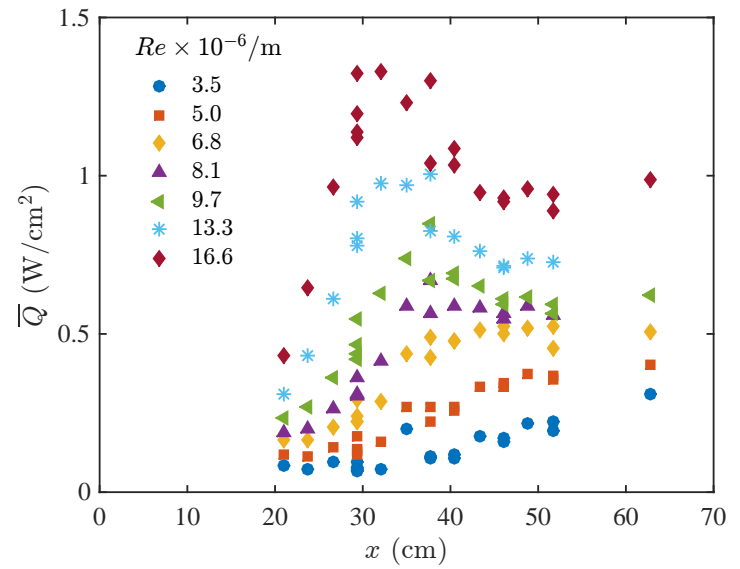

(a) Mean heat flux.

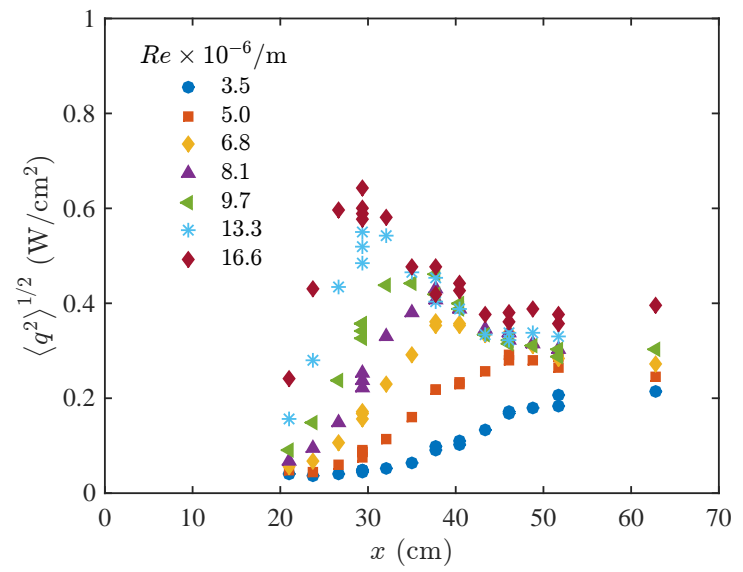

(b) Broadband RMS heat flux.

Figure 5. Mean and broadband RMS heat-flux measurements along the flat plate model centerline for different freestream unit Reynolds numbers. The flat plate model was at $\alpha=0^{\circ}$ for this data set.

where $\omega_{\Psi} \in[5,6]$ is a constant that enforces the admissibility condition. The dilated and translated versions of the mother wavelet take the form:

$$
\Psi_{a, \tau}(t)=a^{-1 / 2} \Psi\left(\frac{t-\tau}{a}\right)
$$

where $a>0$ denotes the scale and $\tau$ is the time delay. The normalization by $a^{-1 / 2}$ ensures that all dilated versions of the mother wavelet have the same energy. The Morlet wavelet was chosen because, in the frequency domain, dilated and translated wavelets can be viewed as constant percentage bandpass filters; i.e., $\Delta_{f} / f=$ const. Thus, a continuous wavelet transform using the Morlet wavelet provides a measure of how the frequency content of the signal evolves in time. The algorithm provided by Jordan et al. ${ }^{22}$ was used to calculate the continuous wavelet transform for our discrete time series.

\section{Results}

Time series data from the ALTP sensors were acquired over a range of freestream unit Reynolds numbers from $3.5 \times 10^{6} \mathrm{~m}^{-1}$ to $16.4 \times 10^{6} \mathrm{~m}^{-1}$. Most of the data were collected with the flat-plate model at zero angle of attack, $\alpha=0.0^{\circ} \pm 0.2^{\circ}$. A subset of data was also acquired with the flat plate at $\alpha=-5^{\circ} \pm 0.2^{\circ}$. In that case, the oblique shock on the top surface of the flat plate reduced the boundary-layer edge Mach number to $\approx 5.3$. For both model angles, the ratio of the wall temperature to the adiabatic wall temperature, $T_{w} / T_{a w}$, ranged from 0.67 to 0.72 .

The mean and broadband root-mean-square (RMS) heat flux for all sensor locations and all of the unit Reynolds numbers considered with the flat plate at $\alpha=0^{\circ}$ are shown in Figs. 5a and 5b. The broadband RMS heat flux was obtained by integrating the auto-spectral density over a frequency range from $100 \mathrm{~Hz}$ to $900 \mathrm{kHz}$. For the lowest unit Reynolds number, $3.5 \times 10^{6} \mathrm{~m}^{-1}$, both the mean and RMS heat flux are observed to increase with increasing distance downstream of $x \approx 30 \mathrm{~cm}$. This is presumably due to the unstable laminar boundary layer developing along the flat plate. As the unit Reynolds number is increased, the streamwise location at which these quantities increase moves upstream. For still higher unit Reynolds numbers, both the mean and RMS heat flux peak and then decay with increasing downstream position. This behavior indicates the onset of breakdown and transition to turbulence. For some of the streamwise positions shown in the plots, heat-flux data were acquired with several different sensors during different runs of the wind tunnel. For example, four separate measurements were made at $x=29.3 \mathrm{~cm}$. Here, the mean heat flux readings varied by $10 \%$ to $24 \%$ and the broadband RMS heat-flux readings varied by $4 \%$ to $10 \%$, depending on the unit Reynolds number at which the data were acquired. In part, this variation is due to the uncertainty in the static sensitivity of the ALTP sensors, which the manufacturer states can be as high as $\pm 20 \% .{ }^{23}$ But it is also likely impacted by how flush the ALTP sensor plug is to the surface of the flat-plate 


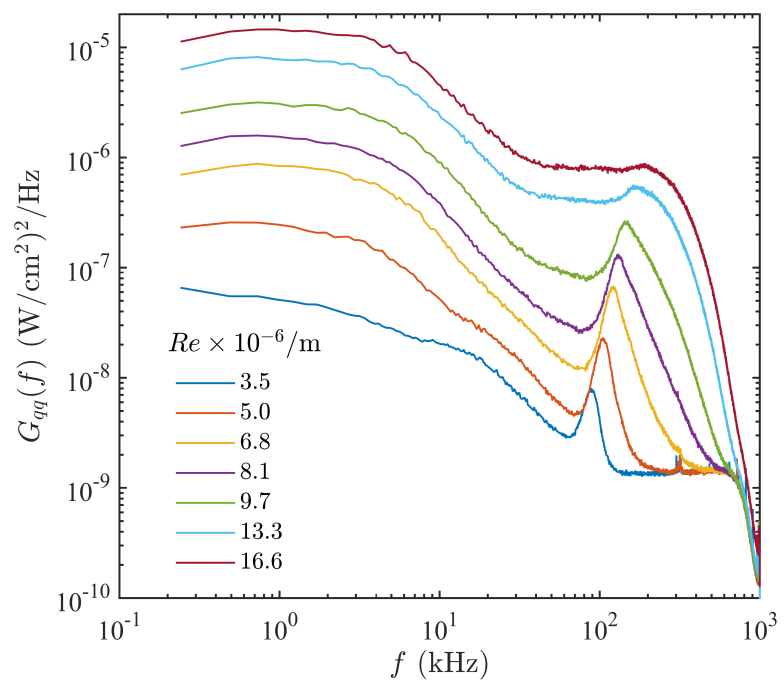

(a) Heat flux auto-spectral densities at $x=26.54 \mathrm{~cm}$ for a range of freestream unit Reynolds numbers.

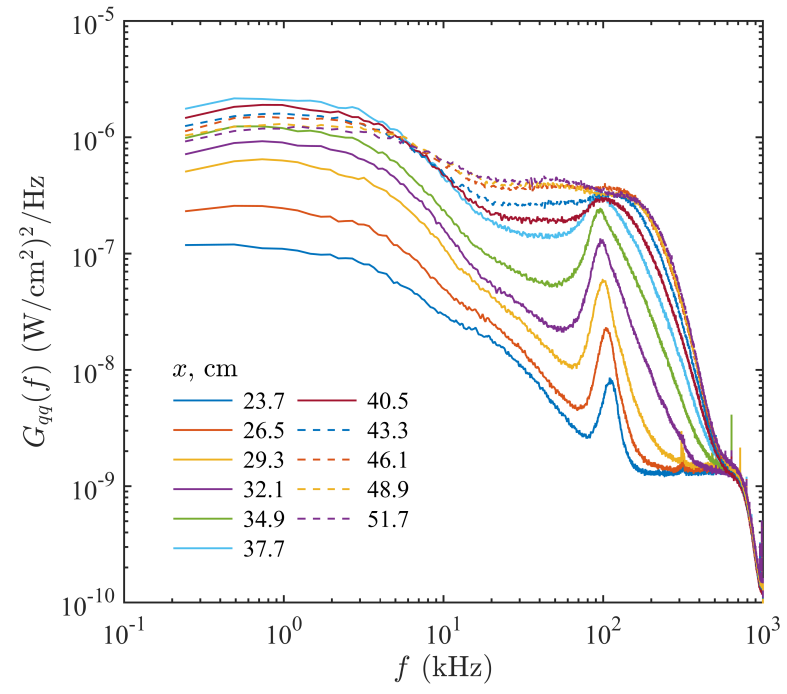

(b) Streamwise evolution of heat flux auto-spectral density at a freestream unit Reynolds number of $5.0 \times 10^{6} \mathrm{~m}^{-1}$.

Figure 6. Measured heat-flux auto-spectral densities on the centerline of the flat-plate model at $\alpha=0^{\circ}$.

model for a given sensor installation.

Sample heat-flux auto-spectral densities acquired with the model at $\alpha=0^{\circ}$ are shown in Fig. 6. In Fig. 6a, heat-flux auto-spectral densities at a fixed streamwise location of $x=26.54 \mathrm{~cm}$ are shown for several different unit Reynolds numbers, while in Fig. 6b, heat-flux auto-spectral densities for different streamwise positions along the flat plate centerline are shown for a single unit Reynolds number of $5.0 \times 10^{6} \mathrm{~m}^{-1}$. In general, the spectra display a narrow band of frequencies, on the order of $100 \mathrm{kHz}$, that grow in amplitude with either increasing unit Reynolds number or increasing streamwise position. On the basis of linear stability calculations for our test conditions, this unstable band of frequencies is associated with the second-mode instability of a hypersonic flat plate laminar boundary layer. The observed shift in the peak frequency of the second-mode mode instability follows the well known inverse scaling of second-mode frequencies with the boundary-layer thickness; i.e., $f \propto u_{e} / 2 \delta$ where $u_{e}$ is the boundary-layer edge velocity and $\delta$ is the boundary-layer thickness. ${ }^{1}$ In Fig. 6a, the increase in the most unstable second-mode frequency with increasing Reynolds number is due to the decreasing boundary-layer thickness at this $x$ location. Likewise, in Fig. 6b, the decrease in the most unstable second-mode frequency with increasing streamwise position is due to the boundary-layer growth. In both figures, spectral broadening about the most unstable secondmode frequency is observed as the flow moves toward breakdown to turbulence. It is interesting to note that harmonics of the second-mode instability do not appear in the spectra. Previous measurements on planar geometries, both off body and on surface, also found this to be the case. ${ }^{15,24}$ This is in contrast to measurements on conical geometries, where harmonics of the second-mode instability are observed. ${ }^{13,24}$ Another point of interest is the significant growth of low-frequency energy (for frequencies $<10 \mathrm{kHz}$ ). Again, that was observed in previous stability measurements on planar geometries, but the exact mechanism for this low-frequency energy growth is unknown. ${ }^{15,24}$

Heat-flux auto-spectral densities acquired on the flat plate model at $\alpha=0^{\circ}$ and $\alpha=-5^{\circ}$ for a freestream unit Reynolds number of $3.5 \times 10^{6} \mathrm{~m}^{-1}$ are shown in Figs. 7a and 7b. In both cases, an unstable band of frequencies around that expected for a second-mode instability (on the order of $100 \mathrm{kHz}$ ) is observed to grow with increasing streamwise position on the model. However, there are several important differences between the two angle cases. First, the peak frequencies of the unstable second-mode band increase as the model is pitched down from $\alpha=0^{\circ}$ to $\alpha=-5^{\circ}$. Since the edge Mach number decreases and the Reynolds number increases across the oblique shock that is generated at $\alpha=-5^{\circ}$, the boundary-layer thickness, which is proportional to $M_{e}^{2} x / \sqrt{R e_{x}}$, decreases. In turn, the second-mode frequencies, which are inversely proportional to $\delta$, are increased. Second, the growth rates of the second-mode disturbances appear to decrease as the model is pitched up from $\alpha=-5^{\circ}$ to $\alpha=0^{\circ}$. That difference is consistent with linear stability theory, which indicates that second-mode growth rates decrease with increasing hypersonic edge Mach numbers. ${ }^{25}$ 


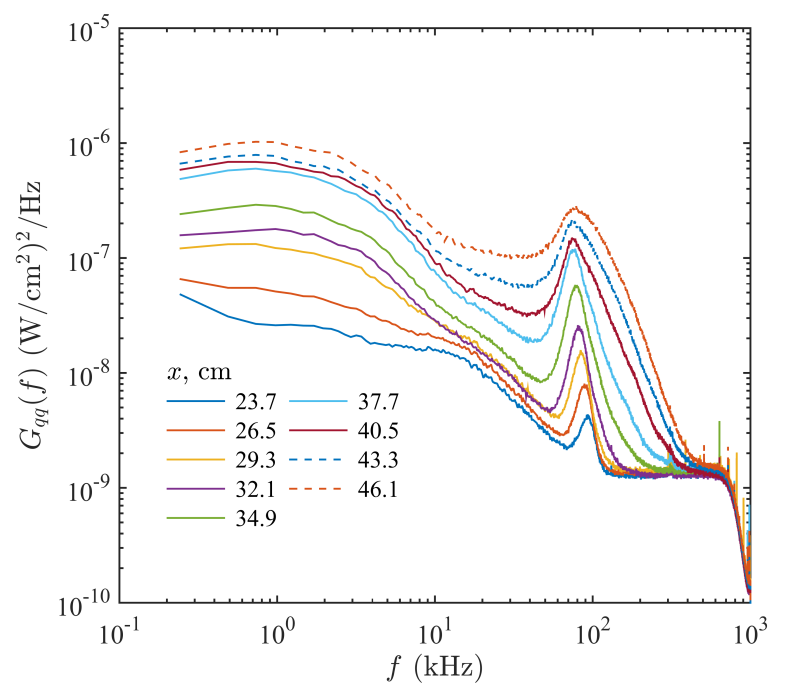

(a) Streamwise evolution of heat flux auto-spectral density at $R e=3.5 \times 10^{6} \mathrm{~m}^{-1}$ and $\alpha=0.0^{\circ}$.

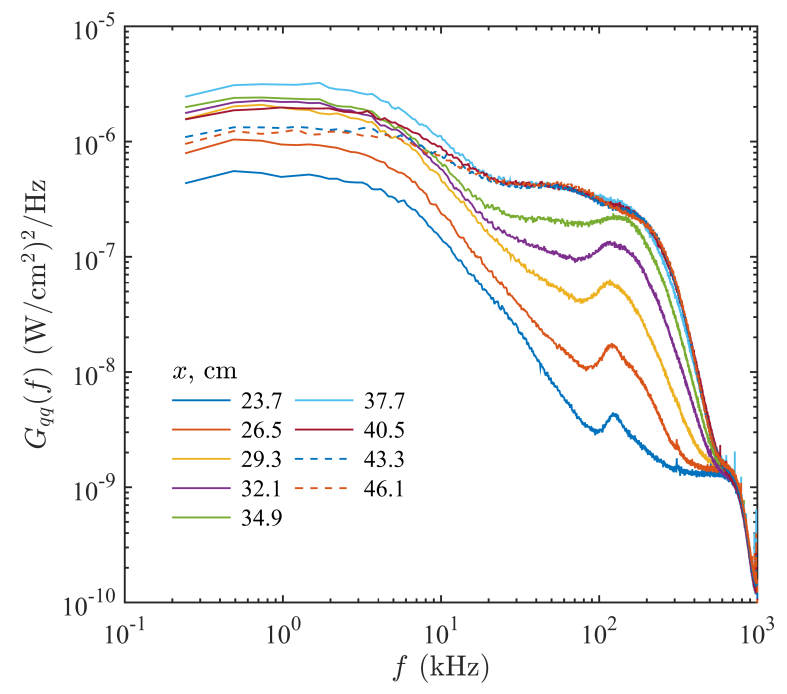

(b) Streamwise evolution of heat flux auto-spectral density at $R e=3.5 \times 10^{6} \mathrm{~m}^{-1}$ and $\alpha=-5.0^{\circ}$.

Figure 7. Comparison of heat-flux auto-spectral densities on the centerline of the flat-plate model at $\alpha=0.0^{\circ}$ and $\alpha=-5.0^{\circ}$.

Third, for the $\alpha=-5^{\circ}$ case, the disturbance growth over the measurement region is large enough to bring about breakdown to turbulence, as evidenced by the broad spectral character for streamwise positions beyond $x=34.9 \mathrm{~cm}$. In contrast, for the $\alpha=0^{\circ}$ case, a distinct unstable band of second-mode frequencies is still observed up to the last measurement station and breakdown to turbulence has not occurred. In this case, as the model angle is increased from $\alpha=-5^{\circ}$ to $\alpha=0^{\circ}$, the Reynolds number, $R=\sqrt{R e_{x}}$, decreases and the edge Mach number increases, both factors acting to move the transition location downstream. Finally, the spectral energy at relatively low frequencies $(<10 \mathrm{kHz})$ is larger for the $\alpha=-5^{\circ}$ case, and the trend in energy growth is different for the two cases. For the $\alpha=-5^{\circ}$ case, there is initial energy growth, followed by saturation and then decay as breakdown to turbulence proceeds with increasing streamwise position. For the $\alpha=0^{\circ}$ case, there is energy growth up to the last measurement station, but breakdown to turbulence does not occur. As stated above, however, the exact mechanism for the low-frequency energy growth in either case is unknown.

The peak frequencies of the second-mode disturbances were extracted from the heat flux auto-spectral density estimates and are plotted as a function of $u_{e} R /\left(M_{e}^{2} x\right)$ in Fig. 8. Here, the abscissa parameter is proportional to $u_{e} / \delta$. Frequency data for a range of unit Reynolds numbers are shown for $\alpha=0^{\circ}$, and at each unit Reynolds number, frequency data for multiple streamwise positions are included. As observed in the plot, the frequencies fall along a straight line, corroborating the proportional scaling with $u_{e} / \delta$. Peak frequencies for the $\alpha=-5^{\circ}$ case at $R e=3.5 \times 10^{6} \mathrm{~m}^{-1}$ are also shown. Accounting for changes in the edge Mach number and Reynolds number across the oblique shock, that data also falls along the same straight line.

To get a sense of the repeatability in the spectral measurements, heat-flux auto-spectral densities as measured with different ALTP sensors at a single streamwise station are shown in Fig. 9. In this example plot, the data were acquired at a streamwise station of $x=29.34 \mathrm{~cm}$, with $R e=3.5 \times 10^{6} \mathrm{~m}^{-1}$ and $\alpha=0.0^{\circ}$. Two of the auto-spectral densities shown were acquired with the same ALTP sensor (sensor number 1) during separate runs of the wind tunnel. Between these runs, the sensor plug was removed from the model and then reinstalled to assess whether our installation procedure influenced the measurements. In general, the auto-spectral densities shown in Fig. 9 have the same shape, but there are differences in the amplitude. In fact, the broadband RMS (100 Hz to $900 \mathrm{kHz}$ ) that is obtained by integrating the auto-spectra varies by $7 \%$. However, for the repeat measurements with sensor number 1, the spectral amplitude is nearly the same, with the broadband RMS differing by less than $1 \%$. This suggests that our installation procedure is consistent for a given sensor. Nevertheless, there is still potential for measurement variability from one sensor to another, particularly if a given sensor is not perfectly flush to the model surface. The other factor that affects the 


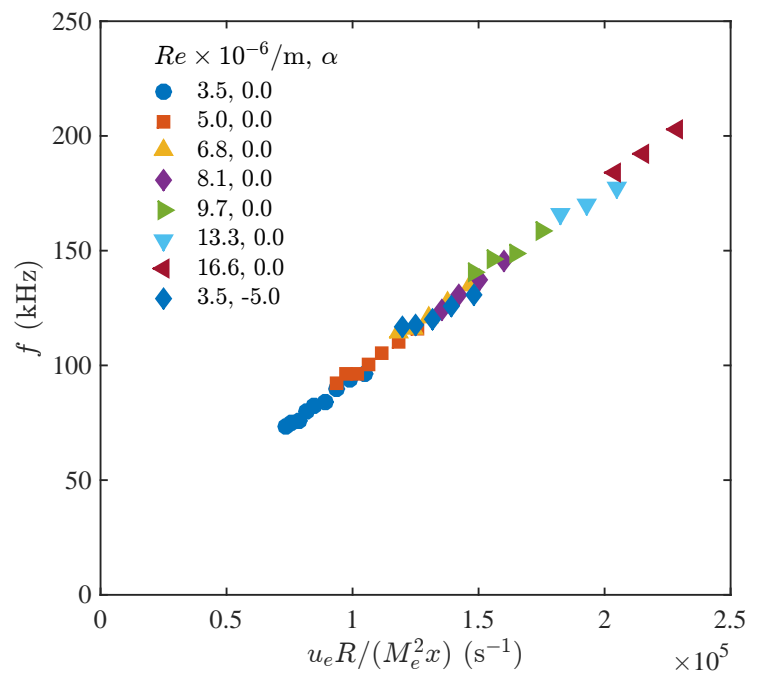

Figure 8. Measured frequencies of the most unstable second-mode disturbances on the flat-plate model. Note that the abscissa parameter, $\mathbf{u}_{\mathbf{e}} \mathbf{R} /\left(\mathbf{M}_{\mathbf{e}}^{2} \mathbf{x}\right)$, is proportional to $\mathbf{u}_{\mathbf{e}} / \delta$.

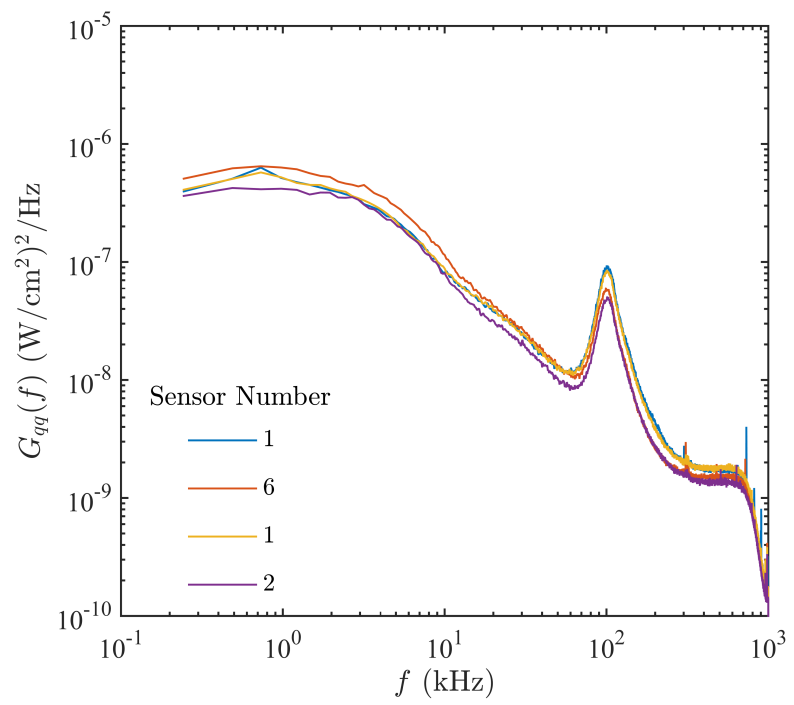

Figure 9. Heat flux auto-spectral densities as measured with different ALTP sensors at a streamwise location of $\mathrm{x}=29.34 \mathrm{~cm}$. $\operatorname{Re}=3.5 \times 10^{6} \mathrm{~m}^{-1}, \alpha=0.0^{\circ}$.

sensor-to-sensor measurement variability is the uncertainty in the sensor static sensitivity, which as stated earlier, could be as large as $\pm 20 \%$.

Sample heat flux time series at several streamwise stations are shown in Fig. 10. Here, the time-series data for the four sensors were acquired simultaneously at a freestream unit Reynolds number of $8.1 \times 10^{6} \mathrm{~m}^{-1}$ and $\alpha=0^{\circ}$. The plotted time series were digitally bandpass filtered (from 70 to $200 \mathrm{kHz}$ ) about the most unstable second-mode disturbance to more clearly observe the instability-wave characteristics. The wave-packet nature of the second-mode instability waves, and their growth and propagation with increasing downstream position, is clearly observed. Similar observations have been made in previous experimental studies of second-mode instabilities, with both off-body ${ }^{26,27}$ and surface measurements ${ }^{15}$ displaying the wave-packet nature of the instabilities. 

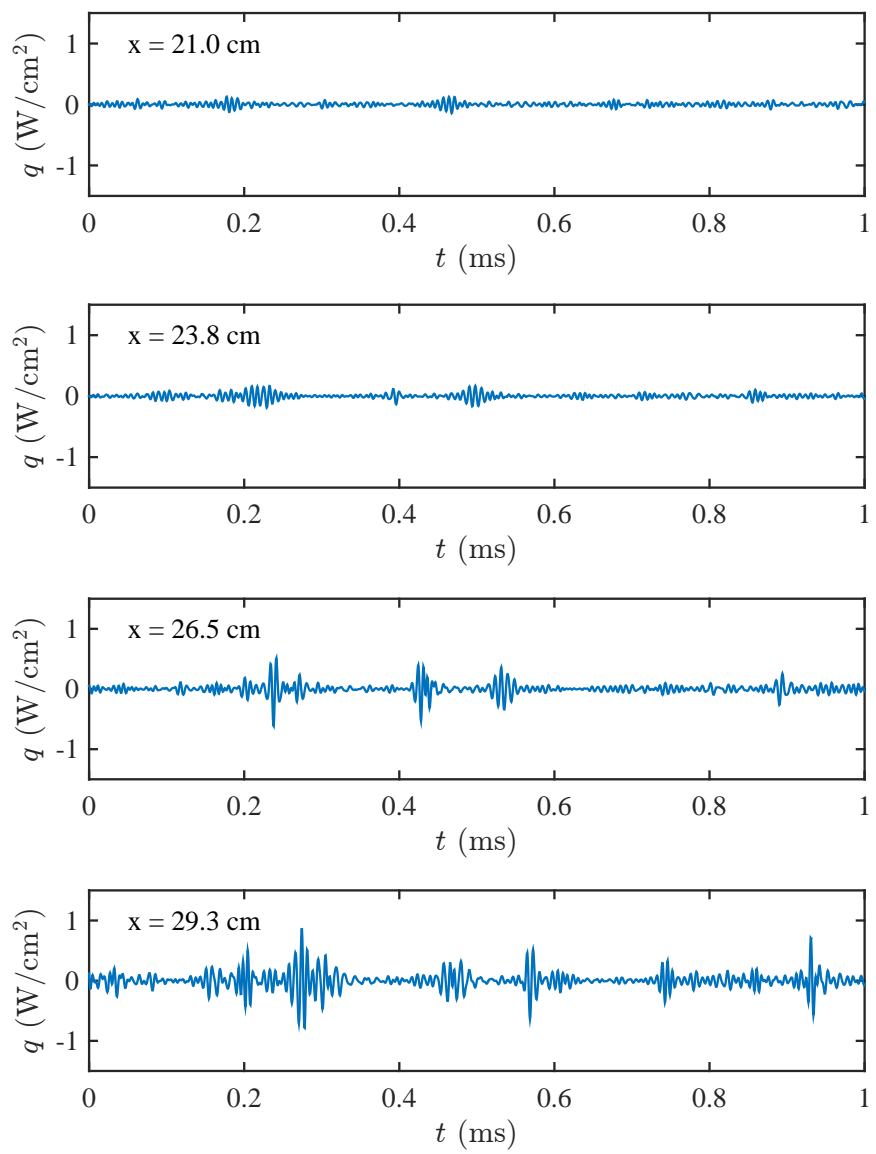

Figure 10. Heat flux time series at several streamwise positions. The time series shown were acquired simultaneously during a run and digitally bandpass filtered (from 70 to $200 \mathrm{kHz}$ ) about the most unstable second-mode disturbance. $R e=8.1 \times 10^{6} \mathrm{~m}^{-1}$. The flat plate model was at $\alpha=0^{\circ}$ for this data set.

To estimate the group velocity of these second-mode wave packets, the cross-correlation coefficients between adjacent heat-flux sensors were calculated for these and similar bandpass filtered time series. The cross-correlation coefficient was defined as: ${ }^{28}$

$$
\rho(\tau)=\frac{R_{q_{i} q_{i+1}}(\tau)}{\sqrt{R_{q_{i} q_{i}}(0)} \sqrt{R_{q_{i+1} q_{i+1}}(0)}}
$$

where $R_{q_{i} q_{i+1}}(\tau)$ is the cross-correlation function between heat-flux sensors at ports $i$ and $i+1, \tau$ is the lag time, and $R_{q_{i} q_{i}}(0)$ and $R_{q_{i+1} q_{i+1}}(0)$ are the auto-correlation functions of each sensor at zero lag time. The values of the cross-correlation coefficient are bounded between \pm 1 . Example cross-correlation coefficients for a unit Reynolds number of $5.0 \times 10^{6} \mathrm{~m}^{-1}$ and $\alpha=0.0^{\circ}$ are shown in Fig. 11. Here, the cross-correlation coefficients are limited in time duration due to the wave-packet nature of the second-mode instabilities and they oscillate at the characteristic frequency of the second-mode disturbances. The propagation time of the wave packets from one sensor to the next is provided by the lag time at which the cross-correlation coefficient peaks, which for the example data shown, occurs at a lag time of $0.034 \pm 0.001 \mathrm{~ms}$ for all of the streamwise positions. Using the peak lag time and the spacing between adjacent sensors $(\Delta x=2.79 \mathrm{~cm})$, the group velocity for the second-mode wave packets was calculated to be $c_{g}=821 \mathrm{~m} / \mathrm{s}$ or $c_{g} / u_{e}=0.87$. The same value for the group velocity was calculated for all of the unit Reynolds numbers considered at $\alpha=0.0^{\circ}$. This 


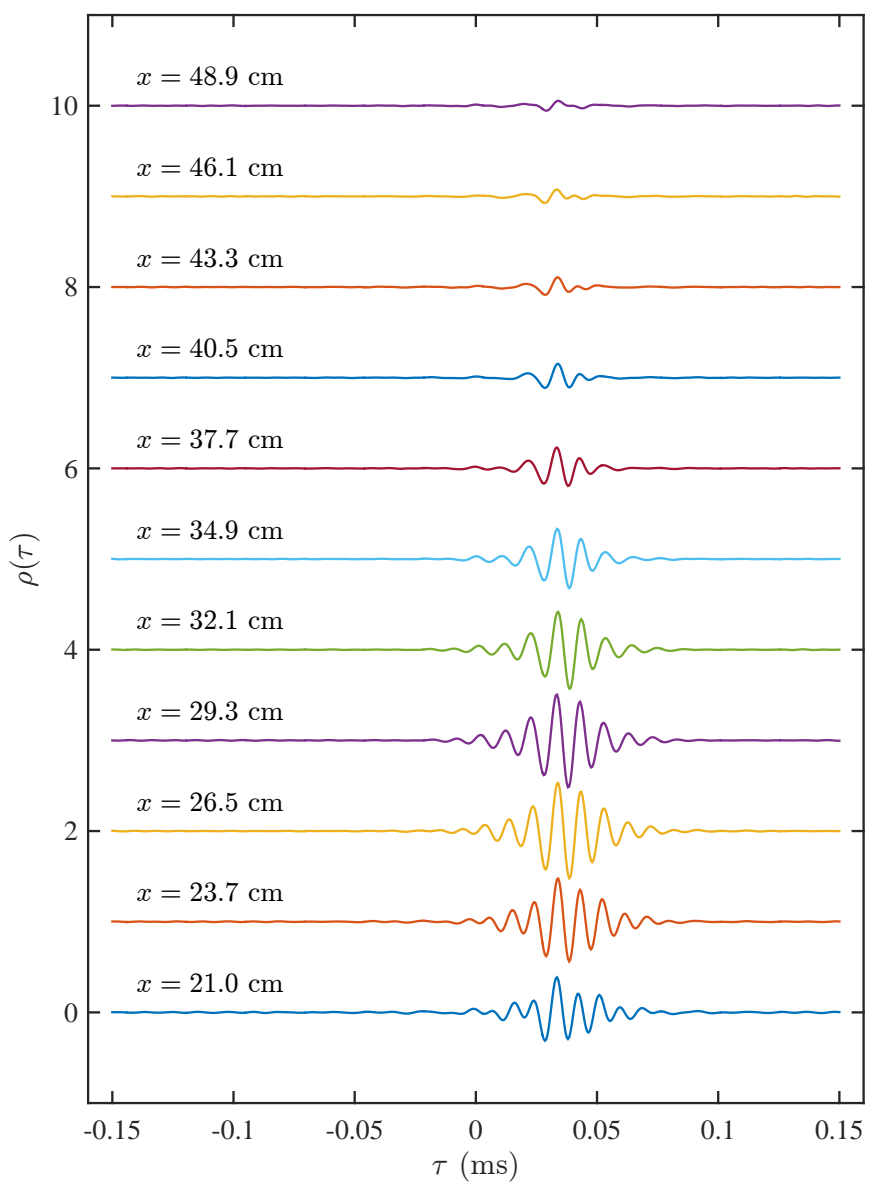

Figure 11. Cross-correlation coefficient between adjacent ALTP sensor pairs. The annotated x-location for each line denotes the location of the upstream sensor and the second sensor is $\Delta x=2.79 \mathrm{~cm}$ downstream. $\operatorname{Re}=5.0 \times 10^{6} \mathrm{~m}^{-1}, \alpha=0.0^{\circ}$. Each curve in the plot was shifted by one unit relative to the previous one for clarity.

group velocity is in good agreement with the second-mode propagation speed recently reported by Heitmann et al. ${ }^{15}$ where ALTP sensors were also used for their measurements. Note that an implicit assumption in the calculation of the group velocity is that the instability waves are two dimensional. This is a reasonable assumption given that stability theory for second-mode instabilities indicates that 2-D waves are the most amplified and previous experimental measurements support that assertion. ${ }^{27}$ It is also of interest to note the decrease of the cross-correlation coefficient, both in terms of peak value and lag duration, that occurs with increasing downstream position. This loss of correlation between sensor pairs occurs as the boundary layer progresses towards breakdown to turbulence, where the wave-packet nature of the time series is lost and the signal becomes more broadband in character.

In Fig. 12, the measured phase speeds between adjacent sensor pairs are shown for a unit Reynolds number of $5.0 \times 10^{6} \mathrm{~m}^{-1}$ and $\alpha=0.0^{\circ}$. In the figure legend, the streamwise positions, $x$, correspond to the upstream sensor of the pair used to calculate the phase speed. The frequency range shown in the figure is centered on the band of unstable second-mode frequencies that exist for these flow conditions and positions. As with the calculation of the group velocity, the instability waves are assumed to be 2-D and therefore we limited our phase-speed calculations to frequencies centered on the most unstable second-mode band. For the data shown in the figure, the phase speed varies somewhat with frequency, but it is within $\pm 5 \%$ of the 


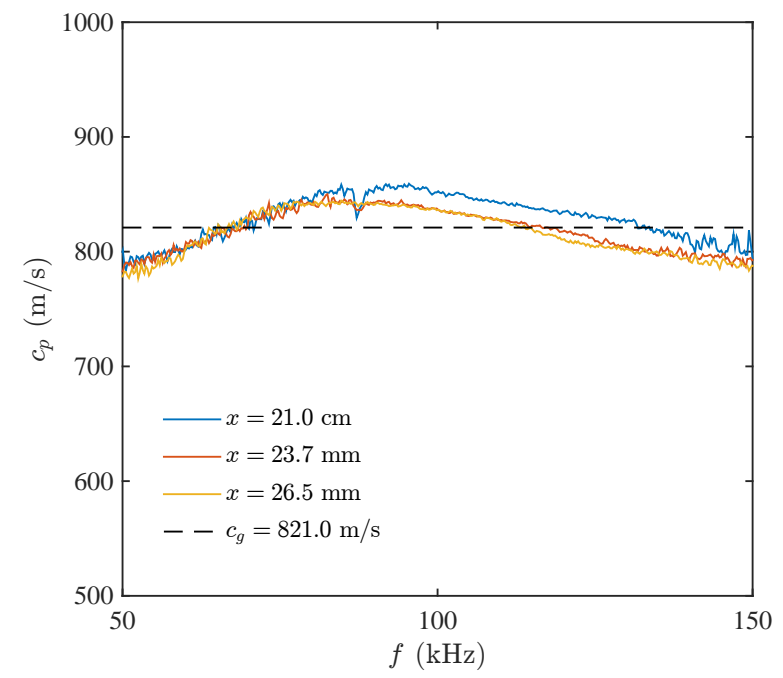

Figure 12. Measured phase speeds for second-mode disturbances at three streamwise locations and Re $=$ $5.0 \times 10^{6} \mathrm{~m}^{-1}, \alpha=0.0^{\circ}$. The dashed line denotes the group velocity as derived from cross correlations at the same streamwise positions.

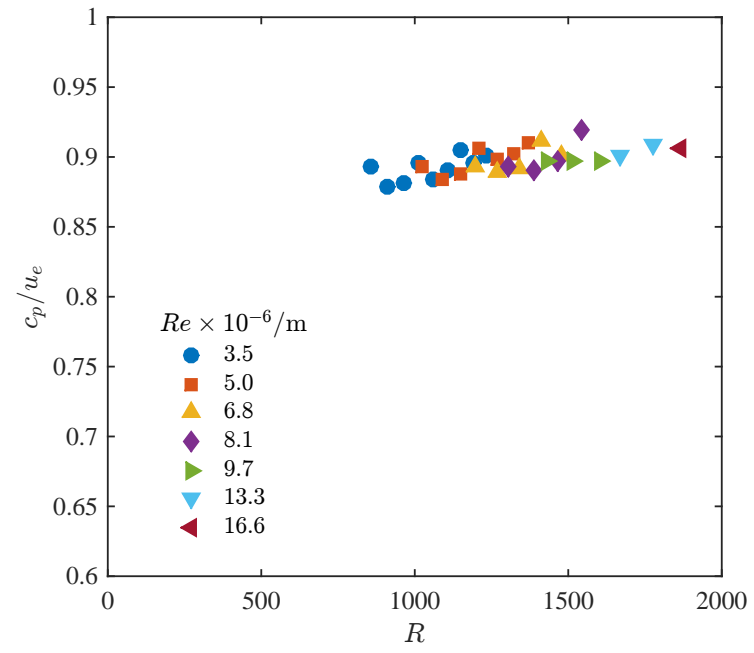

(a) Measured phase speed of the most unstable second-mode disturbances.

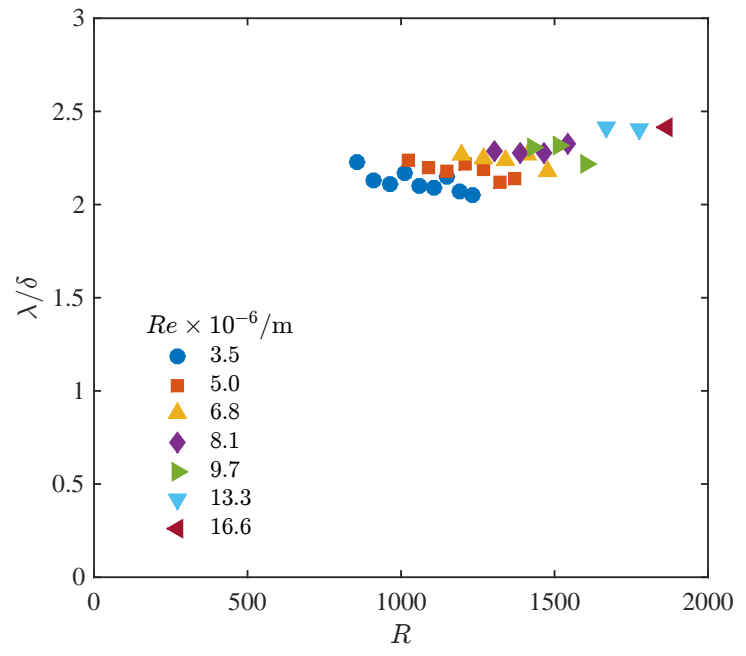

(b) Measured wavelength of the most unstable second-mode disturbances.

Figure 13. Measured phase speed and wavelength of the most unstable second-mode disturbances for a range of freestream unit Reynolds numbers and $\alpha=0.0^{\circ}$.

measured group velocity (represented by the black dashed line in the figure). This indicates that the wave dispersion for the second-mode instabilities is relatively small for this case.

Using phase-speed measurements like that shown in Fig. 12, the phase speeds at the frequencies of the most-unstable second-mode disturbances were extracted and plotted as a function of Reynolds number in Fig. 13a. Here, the phase speeds were normalized by the edge velocity and data are shown for a range of unit Reynolds numbers at $\alpha=0.0^{\circ}$. In general, the phase speeds for the most-unstable second-mode disturbances are roughly $90 \%$ of the boundary layer edge velocity. Using the measured phase speed and frequency of the most-unstable second-mode disturbances, the disturbance wavelengths were calculated and are shown in 
Fig. 13b. Here, the disturbance wavelengths were normalized by the boundary-layer thickness, which was derived from a similarity solution for a laminar boundary layer at our test conditions. In general, the mostunstable second-mode disturbance wavelengths are roughly 2 to 2.5 times the boundary-layer thickness, with a slight increase in wavelength as the Reynolds number is increased. Both the measured phase speeds and disturbance wavelengths reported here are consistent with values previously measured with hot-wires. ${ }^{1,26}$

Sample results for the Morlet-wavelet analysis of the heat flux time series acquired at $R e=5.0 \times 10^{6} \mathrm{~m}^{-1}$ and $\alpha=0.0^{\circ}$ are shown in Figs. 14 to 17. Four different streamwise stations are presented in the figures and it should be noted that the data for each figure were acquired during a different wind tunnel run. In each figure, a $2 \mathrm{~ms}$ segment of the heat flux time series is shown, along with a color contour plot of the magnitude of the Morlet-wavelet transform corresponding to that time-series segment. To aid the interpretation of the wavelet transform, the results are plotted versus frequency, rather than the wavelet scale. The wavelettransforms presented here offer us a view of the time evolution in the frequency content of the time series, and therefore we can identify the frequencies associated with specific events in the time series.

Figure 14 presents the Morlet-wavelet transform for heat flux time series acquired at $x=26.5 \mathrm{~cm}$ from the model leading edge. Here, we observe peaks in the wavelet transform, around a frequency of $100 \mathrm{kHz}$, that occur randomly in time and are of limited time duration. The dashed white line in the figure at a frequency of $105.2 \mathrm{kHz}$, corresponds to the most amplified second-mode instability that was identified in the auto-spectral density for this streamwise position. Therefore, these peaks are associated with the second-mode wave packets observed in the heat flux time series. At this streamwise position, where the second-mode instability is still growing, the frequency band associated with the wave packets is generally narrow. Occasionally, however, a given wave packet will contain a broad range of frequencies $(e . g$., at $t \approx 1.05$ $\mathrm{ms}$ ). The wavelet transform also exhibits large, modulated amplitudes at frequencies below approximately 10 $\mathrm{kHz}$. That energy shows up as a broad, low-frequency band in the ensemble-averaged auto-spectral density (see Fig. 6b).

Moving further downstream to $x=29.3 \mathrm{~cm}$ (Fig. 15), the wavelet transform again exhibits peaks that occur randomly in time, have a short time duration, and have a narrow frequency band centered on the most amplified second-mode frequency $(f=100.6 \mathrm{kHz})$. However, the amplitude of these peaks has grown and that is a reflection of the larger-amplitude wave packets in the heat flux time series. The low-frequency energy is still present at this streamwise station and has also grown in amplitude.

At a streamwise station of $x=37.7 \mathrm{~cm}$ (Fig. 16), we still observe peaks in the wavelet transform that are associated with the second-mode wave packets in the heat flux time series $(f=92.04 \mathrm{kHz})$. But there are other, more frequent events that carry energy at frequencies higher than that of the second-mode frequency. We also observe more intermittent events at mid-band frequencies below the second-mode frequency, but above the low-frequency energy observed at the two previous streamwise stations. The appearance of these high- and mid-frequency events manifests as spectral broadening in the ensemble-averaged auto-spectral density (see Fig. 6b).

Finally, at a streamwise station of $x=46.1 \mathrm{~cm}$ (Fig. 17), there are no longer peaks in the wavelet transform that can be associated with second-mode wave packets. Instead, a broad range of frequencies are represented in the wavelet transform which suggests that breakdown to turbulence is occurring. This is supported by the auto-spectral density at this streamwise station, which displays a broadband spectral character (see Fig. 6b).

\section{Conclusions}

The main objective of the present work was to gain experience with and to determine the suitability of the ALTP heat-flux sensors for transition measurements in our conventional hypersonic blow-down tunnels at NASA Langley Research Center. As a test bed, we instrumented the centerline of a flat plate model with a streamwise array of ALTP sensors and exposed the model to a Mach 6 freestream over a range of unit Reynolds numbers.

Auto-spectral analysis of the heat flux time series revealed an unstable band of frequencies that are associated with the second-mode instability of a hypersonic laminar boundary layer. The frequencies of the most-unstable second-mode disturbance were found to be proportional to $u_{e} / \delta$, as expected. Cross correlations and cross-spectral densities between adjacent heat-flux sensors were calculated, and from those calculations, estimates for the group velocity and phase speed of the second-mode instability waves were obtained. The group velocity was found to be $c_{g} / u_{e}=0.87$ and the phase velocity of the most unstable 

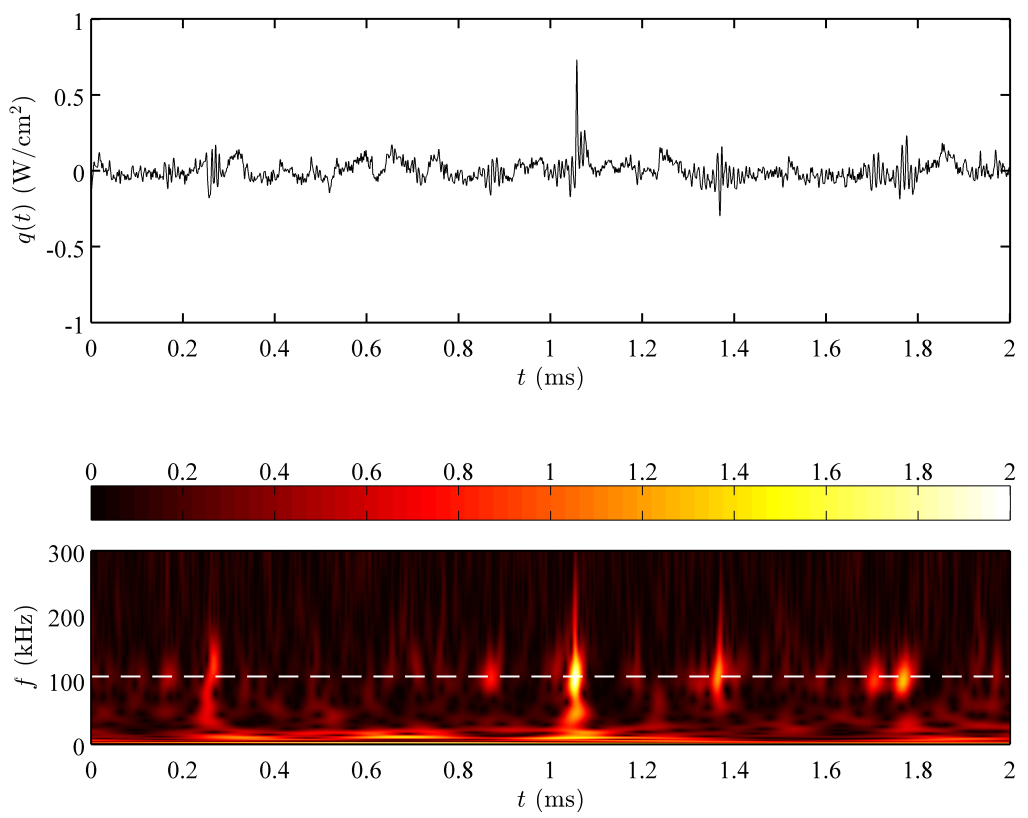

Figure 14. Heat flux time series (top) and the corresponding continuous wavelet transform (bottom). The colors in the contour plot denote the amplitude of the continuous wavelet transform and the white dashed line denotes the frequency of the most unstable second-mode disturbance $(f=105.2 \mathrm{kHz})$. The heat-flux sensor was at $\mathrm{x}=26.5 \mathrm{~cm}$ and $\mathrm{Re}=5.0 \times 10^{6} \mathrm{~m}^{-1}, \alpha=0.0^{\circ}$.
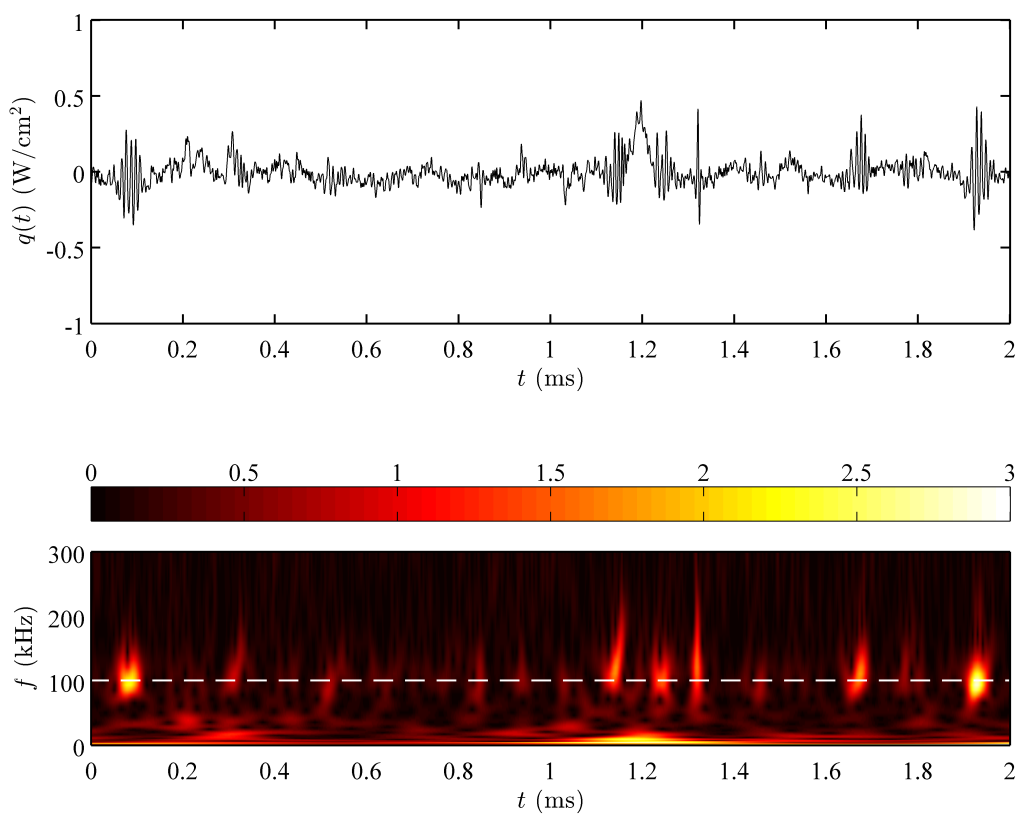

Figure 15. Heat flux time series (top) and the corresponding continuous wavelet transform (bottom). The colors in the contour plot denote the amplitude of the continuous wavelet transform and the white dashed line denotes the frequency of the most unstable second-mode disturbance $(\mathrm{f}=100.6 \mathrm{kHz})$. The heat-flux sensor was at $\mathrm{x}=29.3 \mathrm{~cm}$ and $\mathrm{Re}=\mathbf{5 . 0} \times 10^{6} \mathrm{~m}^{-1}, \alpha=0.0^{\circ}$. 

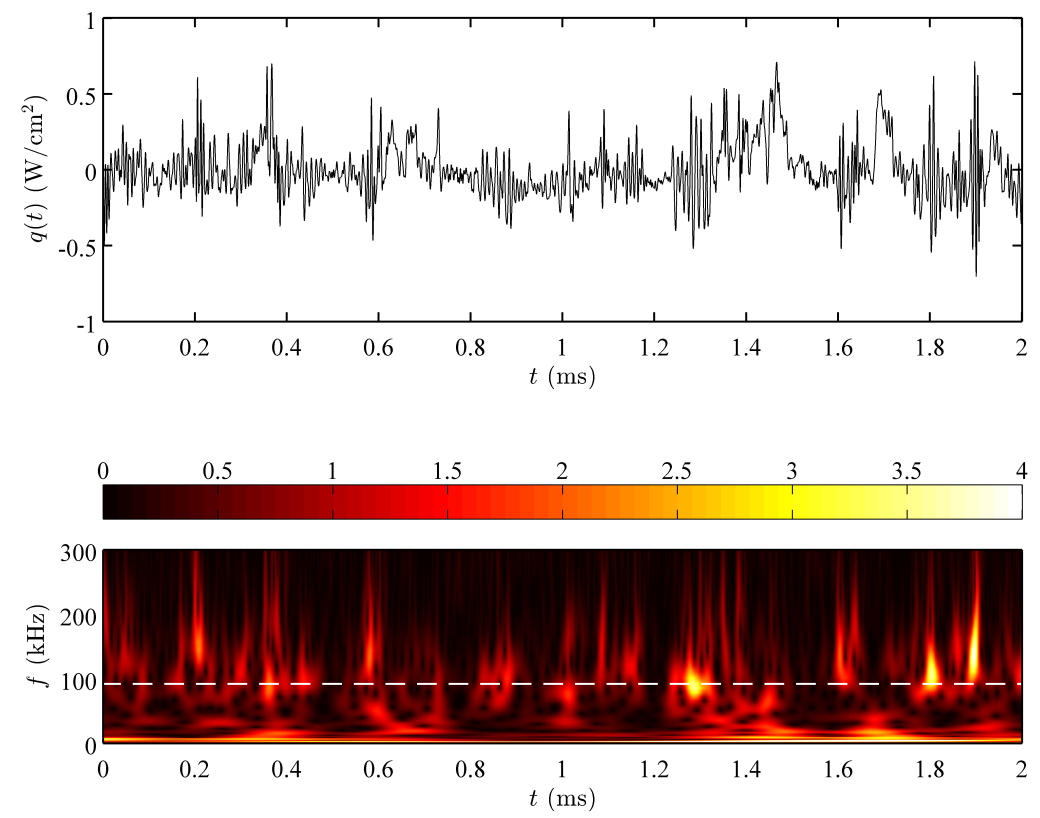

Figure 16. Heat flux time series (top) and the corresponding continuous wavelet transform (bottom). The colors in the contour plot denote the amplitude of the continuous wavelet transform and the white dashed line denotes the frequency of the most unstable second-mode disturbance $(f=92.04 \mathrm{kHz})$. The heat-flux sensor was at $\mathrm{x}=37.7 \mathrm{~cm}$ and $\mathrm{Re}=5.0 \times 10^{6} \mathrm{~m}^{-1}, \alpha=0.0^{\circ}$.
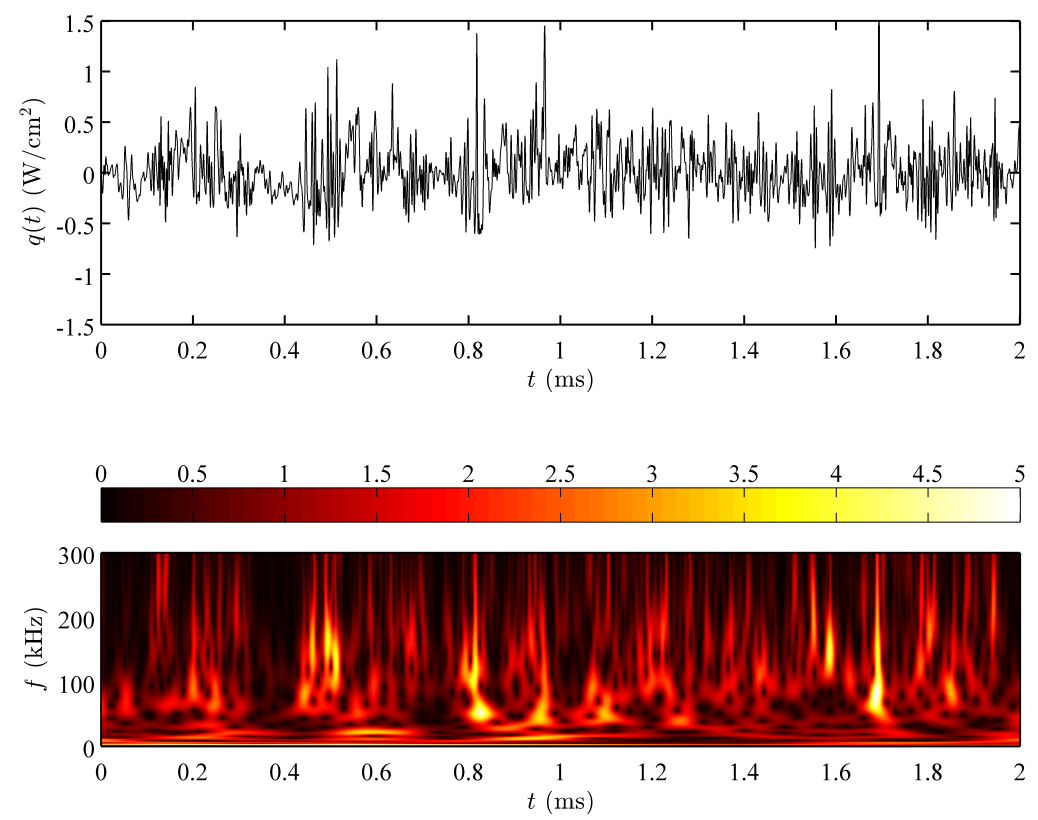

Figure 17. Heat flux time series (top) and the corresponding continuous wavelet transform (bottom). The colors in the contour plot denote the amplitude of the continuous wavelet transform. The heat-flux sensor was at $\mathrm{x}=46.1 \mathrm{~cm}$ and $\mathrm{Re}=5.0 \times 10^{6} \mathrm{~m}^{-1}, \alpha=0.0^{\circ}$. 
frequency was approximately $c_{p} / u_{e} \approx 0.90$. The wavelength of the second-mode instability waves was also calculated and was found to be 2 to 2.5 times the boundary-layer thickness. These second-mode wave parameters are in good agreement with previously published experimental results. Heat flux time series, and the Morlet-wavelet transforms of them, revealed the wave-packet nature of the second-mode instability waves, which is consistent with previous observations. Given the good agreement between the present measurements and previously reported data for second-mode instability waves (from both off-body and surface measurements), we conclude that the ALTP sensors can provide useful quantitative data on various characteristics of transition in a hypersonic boundary layer. Although the ALTP sensor is surface based, and therefore provides only a partial picture of the transition process, it is a good alternative in cases where off-body unsteady measurements in the boundary layer are not feasible.

In this paper, we presented a laser-based radiative heating system that was used to measure the frequency response functions of our ALTP sensors. Pre-test measurements of the FRFs for our sensor batch indicated a flat frequency response with a cutoff frequency of $650 \mathrm{kHz}$. The FRFs for all of the sensors tested were nearly identical. Post-test measurements of the FRFs were found to be essentially the same as the pre-test FRFs, indicating that the sensor dynamic responses were stable over the duration of the wind-tunnel test. In post-test data analysis, the measured sensor FRFs were used to correct spectral estimates for any attenuation caused by the finite sensor bandwidth.

High-bandwidth, quantitative, unsteady measurements are critical to the study of hypersonic boundary layer transition, and to hypersonic fluid mechanics problems in general. As such, it is our hope that the ALTP heat-flux sensor technology will see continued development. To that end, improvements in the sensor packaging and robustness are needed, and a reduction in sensor-package size would greatly aid installation in scale models. Perhaps the weakest aspect of the sensor is the reported uncertainty in the static sensitivity, which led to some variability in our measurements of the mean and instantaneous heat flux. Improvements in the static calibration of the ALTP sensor will need to be developed.

\section{References}

\footnotetext{
${ }^{1}$ Stetson, K. F., "Hypersonic Boundary-Layer Transition," Advances in Hypersonics: Defining the Hypersonic Environment, edited by J. J. Bertin, J. Periaux, and J. Ballmann, Vol. 1, Birkhauser, Boston, MA, 1992, pp. 324-417.

${ }^{2}$ Lachowicz, J. T., Chokani, N., and Wilkinson, S. P., "Boundary-Layer Stability Measurements in a Hypersonic Quiet Tunnel," AIAA Journal, Vol. 34, No. 12, December 1996, pp. 2496-2500.

${ }^{3}$ Hofferth, J. W., Boundary-Layer Stability and Transition on a Flared Cone in a Mach 6 Quiet Wind Tunnel, Ph.D. thesis, Texas A\&M University, College Station, TX, August 2013.

${ }^{4}$ Craig, S. A., Stability of High-Speed, Three-Dimensional Boundary Layers, Ph.D. thesis, Texas A\&M University, College Station, TX, May 2015.

${ }^{5}$ Hofferth, J. W., Humble, R. A., Floryan, D. C., and Saric, W. S., "High-Bandwidth Opitcal Measurements of the Second-Mode Instability in a Mach 6 Quiet Tunnel," AIAA Paper 2013-0378, January 2013.

${ }^{6}$ Parziale, N. J., Shepherd, J. E., and Hornung, H. G., "Differential Interferometric Measurement of Instability in a Hypersonic Boundary Layer," AIAA Journal, Vol. 51, No. 3, March 2013, pp. 750-753.

${ }^{7}$ Fujii, K., "Experiment of the Two-Dimensional Roughness Effect on Hypersonic Boundary-Layer Transition," Journal of Spacecraft and Rockets, Vol. 43, No. 4, July-August 2006, pp. 731-738.

${ }^{8}$ Heitmann, D., Radespiel, R., and Kahler, C., "Investigation of the Response of a Hypersonic 2-D Boundary Layer to Controlled Acoustic Disturbances," AIAA Paper 2010-536, January 2010.

${ }^{9}$ Chynoweth, B. C., Ward, C. A., Greenwood, R. T., McKiernan, G. R., Fisher, R. A., and Schneider, S. P., "Measuring Transition and Instabilities in a Mach 6 Hypersonic Quiet Wind Tunnel," AIAA Paper 2014-2643, June 2014.

${ }^{10}$ Marineau, E. C., Moraru, C. G., Lewis, D. R., Norris, J. D., Lafferty, J. F., Wagnild, R. M., and Smith, J. A., "Mach 10 Boundary-Layer Transition Experiments on Sharp and Blunted Cones," AIAA Paper 2014-3108, June 2014.

${ }^{11}$ Heitmann, D., Kahler, C., Radespiel, R., Roediger, T., Knauss, H., and Kramer, E., "Disturbance-Level and Transition Measurements in a Conical Boundary Layer at Mach 6," AIAA Paper 2008-3951, June 2008.

${ }^{12}$ Knauss, H., Roediger, T., Bountin, D. A., Smorodsky, B. V., Maslov, A. A., and Srulijes, J., "Novel Sensor for Fast Heat-Flux Measurements," Journal of Spacecraft and Rockets, Vol. 46, No. 2, March-April 2009, pp. $255-265$.

${ }^{13}$ Roediger, T., Knauss, H., Estorf, M., Schneider, S. P., and Smorodsky, B. V., "Hypersonic Instability Waves Measured Using Fast-Response Heat-Flux Gauges," AIAA Journal, Vol. 46, No. 2, March-April 2009, pp. 266-273.

${ }^{14}$ Roediger, T., The Atomic Layer Thermopile - A New Heat Transfer Measurement Technique in Fluid Mechanics and Thermodynamics, Ph.D. thesis, University of Stuttgart, Stuttgart, Germany, 2010.

${ }^{15}$ Heitmann, D., Kahler, C., Radespiel, R., Roediger, T., Knauss, H., and Wagner, S., "Non-Intrusive Generation of Instability Waves in a Planar Hypersonic Boundary Layer," Experiments in Fluids, Vol. 50, No. 2, February 2011, pp. $457-464$.

${ }^{16}$ Roediger, T., Knauss, H., Gaisbauer, U., Kraemer, E., Jenkins, S., and von Wolfersdorf, J., "Time-Resolved Heat Transfer Measurements on the Tip Wall of a Ribbed Channel Using a Novel Heat Flux Sensor-Part I: Sensor and Benchmarks," Journal of Turbomachinery, Vol. 130, January 2008, pp. 011018/1-8.
} 
${ }^{17}$ Berger, K. T., Hollingsworth, K. E., Wright, S. A., and Rufer, S. J., "NASA Langley Aerothermodynamic Laboratory: Hypersonic Testing Capabilities," AIAA Paper 2015-1337, January 2015.

${ }^{18}$ Rufer, S. J. and Berridge, D., "Pressure Fluctuation Measurements in the NASA Langley 20-Inch Mach 6 Wind Tunnel," AIAA Paper 2012-3262, June 2012.

${ }^{19}$ Bonnet, J. P. and de Roquefort, T., "Determination and Optimization of Frequency Response of Constant Temperature Hot-Wire Anemometers in Supersonic Flow," Rev. Sci. Instr., Vol. 51, 1980, pp. 234-239.

${ }^{20}$ Kegerise, M. A. and Spina, E. F., "A Comparative Study of Constant-Voltage and Constant-Temperature Hot-Wire Anemometers: Part II - The Dynamic Response," Experiments in Fluids, Vol. 29, 2000, pp. 165-177.

${ }^{21}$ Qian, S. and Chen, D., Joint Time-Frequency Analysis, Prentice Hall, Englewood Cliffs, NJ, 1996.

${ }^{22}$ Jordan, D., Miksad, R. W., and Powers, E. J., "Implementation of the Continuous Wavelet Transform for Digital Time Series Analysis," Rev. Sci. Instr., Vol. 68, No. 3, March 1997, pp. 1484-1494.

${ }^{23}$ ForTech HTS GmbH, Users manual, Ultrafast heat transfer sensor: ALTP, 2011.

${ }^{24}$ Stetson, K. F., Kimmel, R. L., Thompson, E. R., Donaldson, J. C., and Siler, L. G., "A Comparison of Planar and Conical Boundary Layer Stability and Transition at a Mach Number of 8," AIAA Paper 91-1639, June 1991.

${ }^{25}$ Mack, L. M., "Boundary Layer Stability Theory," Rept. 900-277 Rev. A, Jet Propulsion Lab, Pasadena, CA, November 1969.

${ }^{26}$ Stetson, K. F. and Kimmel, R. L., "On Hypersonic Boundary-Layer Stability," AIAA Paper 92-737, January 1992.

${ }^{27}$ Poggie, J. and Kimmel, R. L., "Disturbance Evolution and Breakdown to Turbulence in a Hypersonic Boundary Layer Instantaneous Structure," AIAA Paper 97-556, January 1997.

${ }^{28}$ Bendat, J. S. and Piersol, A., Random Data: Analysis and Measurement Procedures, John Wiley and Sons, New York, NY, 1986. 